\title{
СРАВНЕНИЕ ФУНКЦИОНАЛЬНОСТИ ПРОГРАММ, ИСПОЛЬЗУЕМЫХ ДЛЯ РЕШЕНИЯ ЗАДАЧ ГРАВИРАЗВЕДКИ И МАГНИТОРАЗВЕДКИ
}

\author{
И.В. Геник \\ Горный институт УрО РАН, г. Пермь
}

\begin{abstract}
Аннотация: Статья является продолжением исследования, имеющего целью сравнение проприетарных и свободных программ, используемых в геофизике. В предыдущей статье были охарактеризованы общие проблемы создания программного обеспечения и кратко описано свободное программное обеспечение для решения задач гравиметрии и магнитометрии. В данной статье продолжается сравнение программного обеспечения и сделан переход к анализу функциональности. Рассмотрение выполнено для проприетарных программ, используемых в российских условиях: ГИС INTEGRO, «КОСКАД 3D», СИГMA3D, Zond, Oasis montaj, а также для свободной программы РуGMI. Выделены и охарактеризованы для каждой программы главные функциональные блоки: ввод, анализ и преобразование исходных данных; качественные и количественные методы решения обратных задач; комплексная интерпретация, включая задачи классификации; визуализация исходных данных и результатов.

Установлено, что функциональность проприетарных программ зависит от изначальных намерений авторов: использовать преимущественно для внутреннего употребления или для продажи. Программы, первого направления имеют большее разнообразие интерпретационных методов, но методы зачастую дублируют друг друга и имеют менее развитые сервисные возможности для оперирования с исходными данными (преобразование в другие форматы, интерполяция и др.). Программы, предназначенные для продажи, имеют большие сервисные функции, но меньшие функциональные. Продвинутые средства в этом случае предоставляются за дополнительную плату, весьма немалую, например, для «Oasis montaj». Из свободных программ проанализирована PyGMI, близкая по возможности к проприетарным программам. Программа предназначена для решения широкого круга геофизических задач, включая гравиметрию и магнитометрию. Показано что основные блоки имеют функциональное наполнение на уровне проприетарных программ. Исключение составляет интерпретация гравитационного поля, имеющая недостаточную функциональность, но это может быть компенсировано (поскольку программа свободная), добавлением своих методов или использованием других свободных библиотек для получения нужных функций.
\end{abstract}

Ключевые слова: геофизика, гравиразведка, магниторазведка, интерпретация, программное обеспечение, свободное программное обеспечение.

\section{Введение}

Сравнение возможностей современных программных пакетов и рекомендации по их использованию должны опираться на несколько взаимоувязанных критериев. Они должны включать в себя: степень структурированности пакета программ; возможности интерпретации каждым методом (гравиметрия и магнитометрия) и комплексной интерпретации; наличие развитого графического интерфейса; частоту выхода новых версий и исправлений; объем добавленной функциональности при переходе между мажорными ${ }^{1}$ версиями; возможности обратной связи с разработчиком или возможности пользователя менять что-либо в программе. В зависимости от вида программной лицензии ${ }^{2}$ и области применения программ степень применимости данных критериев может варьироваться. Геофизическое программное обеспечение (ПО) с одной стороны достаточно консервативно, т.к. основные методы и подходы $[1,9-11,14,15]$ были в значительной сформированы уже к началу 1980-х годов, а с другой стороны, старается следовать современным трендам развития ПО [2]. Указанные обстоятельства обусловливают конкуренцию между различными пакетами и повышенное внимание к новым пакетам, которых совсем мало в области геофизики.

\footnotetext{
${ }^{1} \mathrm{https} / / /$ en.wikipedia.org/wiki/Software_versioning

${ }^{2}$ https://en.wikipedia.org/wiki/Software_license
} 


\section{Методы и подходы}

Целью работы является анализ общих вопросов, связанных с критериями оценки программного обеспечения в области геофизики, их применение к программам, связанным с обработкой гравиметрических и магнитометрических данных; определение их основных особенностей, путей совершенствования ПО, обеспечивающих повышение эффективности и гибкости использования, а также раскрытие возможностей современных информационных технологий.

\section{Результаты и обсуждение}

В России существует и используется много программ и пакетов, предназначенных для решения задач гравиметрии и магнитометрии: ГИС INTEGRO (авторы: сотрудники лаборатории Геоинформатики ФГУП ГНЦ РФ ВНИИгеосистем, а затем отделения Геоинформатики ФГБУ «ВНИГНИ») ${ }^{3}$, «КОСКАД 3D» ${ }^{4}$ (авторы: А.А. Никтин, А.В. Петров, А.С.Алексашин), «СИГМА3D» ${ }^{5}$ (авторы: П.С. Бабаянц, Ю.И. Блох, А.А. Трусов), «Zond» ${ }^{6}$ (автор: А.Е. Каминский), «Oasis montaj» ${ }^{7}$ (владелец: Seequent Limited), GelioSMI (автор: А.Ю. Давыденко ${ }^{8}$ ) [5], VECTOR (авторы: В.М. Новоселицкий, В.А. Кутин, М.С. Чадаев, С.Г. Бычков, Г.В. Простолупов, В.В. Антипин) [13], MultAlt ${ }^{9}$ (авторы: Ф.М. Гольцман, Д.Ф. Калинин, Т.Б. Калинина) $[4,6]$, а также реализующие интерпретацию гравиразведки и сейморазведки - GCIS $[2,12]$ (авторы А.И. Кобрунов, А.П. Петровский и др.), GeoVIP (авторы: А.И. Кобрунов, С.Г. Куделин, М.И. Барабанов) $[2,7,8]$.

Программы и программные пакеты, предназначенные для решения задача гравиметрии и магнитометрии, имеют различную организацию, функциональность и графический интерфейс.

Рассмотрим особенности наиболее часто используемых программных пакетов.

ГИС INTEGRO - российская картографическая геоинформационная система с расширенными возможностями для геологических задач; разработка системы 1998 г.

Пакет состоит из нескольких блоков: базового, «Прогноз», «Геофизика», «3Dмоделирование», «Геолкарта», «Скважины».

Базовый блок используется при сборе исходных данных, систематизации и каталогизации данных; оцифровке и привязке карт, профилей, разрезов, каротажных диаграмм; оформлении и печати рабочих и итоговых материалов.

В блоке «Прогноз» решаются задачи районирования территорий по комплексу характеристик: прогноз полезных ископаемых и выделение перспективных территорий для дальнейших работ; выделение блоков по гравимагнитным данным; дешифрирование спектрозональных изображений. В блоке выполняются: анализ качества данных (анализ типов распределений, матрица корреляции свойств, анализ вариабельности и др.); факторный, структурный и регрессионный анализы; районирование и разбиение на группы (методом k-средних, по критериям, голотипная ${ }^{10}$, иерархическая); классификация с обучением, включая дискриминантный анализ.

В блоке «Геофизика» выполняется: обработка двух и трехмерных наборов геофизических данных (волновых и потенциальных полей, МТЗ); используются фильтрации и

\footnotetext{
${ }^{3} \mathrm{http}: / /$ www.gis-integro.ru

${ }^{4} \mathrm{http}: / / \operatorname{coscad} 3 \mathrm{~d} . \mathrm{ru}$

${ }^{5} \mathrm{http}: / /$ sigma3d.com

${ }^{6} \mathrm{http}: / /$ zond-geo.com

${ }^{7} \mathrm{https}: / /$ www.seequent.com/ru/продукты-и-решения/geosoft-oasis-montaj

${ }^{8} \mathrm{http}$ ://gelios-geo.com/wp-content/uploads/2016/08/Materialy-seminara-im.-D.G.-Uspenskogo.pdf

${ }^{9} \mathrm{http}: / /$ www.geolraz.com/page/MultAlt

${ }^{10} \mathrm{http}$ //public.wiki.integro.geosys.ru/index.php/Голотипная_таксономия
} 
статистический анализ; решение прямых и обратных задач гравиметрии и магнитометрии; расчет градиентных характеристик.

Блок «3D-моделирование» предназначен для: трехмерной визуализации и трехмерного геологического моделирования; автоматизированного построения сечений и разрезов и ряд других функция, включая увязку сейсмогеологических разрезов и результатов геологической интерпретации по профилям.

Блок «Геолкарта» используется при: создании цифровых моделей геологических карт и их подготовки к изданию, включая технологии генерализации объектов карты (объединение, сглаживание, упрощение и др.).

Блок «Скважины» дает возможности: хранения и визуализации (2D и 3D) данных по скважинам; отобразить и редактировать данные по скважинам на планшете, на профиле;

Таким образом, ГИС INTEGRO изначально хорошо структурирован на описанные выше блоки. Дополнительно можно отметить: сайт программы имеет русский и английский варианты; имеются демо-версии; подробное руководство пользователя ${ }^{11}$; на youtube.com есть канал, посвященный программе; на сайте имеются обучающие курсы, которые прошли сотрудник 17 крупных российских образовательных, исследовательских и производственных организаций.

Российский пакет «КОСКАД 3D» - комплекс спектрально-корреляционного анализа данных. Первая версия пакета появилась в 1980 г. как результат переноса технологий сейсморазведки для решения задач геопотенциальных полей в области рудной геофизики. Программа неплохо структурирована, в меню имеются пункты: «Сервис», «Графика», «Статистика», «Фильтрация», «Обнаружение», «Комплекс». Рассмотрим их по порядку.

В меню «Сервис» расположены команды для работы с данными: ввод данных из файлов различных форматов, интерполяция и экстраполяция данных, дополнительные преобразования данных (вставка, объединение, вращение и т.д.), работа с сейсмической информацией, а также некоторые настройки.

В меню «Графика» имеются возможности для построения графиков, карт и трехмерных отображений (кубов). Имеются возможности выбора фрагментов полей и диапазонов их просмотра.

В меню «Статистика» расположены команды для: статистического, спектрального, корреляционного анализа; расчета градиентных характеристик геофизических полей; выполнения вейвлет-анализа и оценки анизотропии полей. Расчеты могут выполняться в скользящих окнах разной размерности, позволяя получить различные параметры описательной статистики ${ }^{12}$. Кроме указанных выше методов качественной интерпретации имеются методы количественной интерпретации: прямые задачи 2D и 3D гравиметрии, оценки параметров моделей, глубин гравимагнитных поверхностей (обратные задачи) и трассирование осей аномалий.

В меню «Фильтрация» предлагаются одно-, двух-, трехмерная фильтрации разных типов: в фиксированном окне, в окне «живой» формы, адаптивная и другие.

В меню «Обнаружение» находятся команды, нацеленные на выделение геофизических аномалий линейной и произвольной формы, имеющих малую интенсивность, соизмеримую с уровнем помех.

В меню «Комплекс» выполняется обработка многопризнаковой геологогеофизической информации с использованием методов классификации, распознавания

\footnotetext{
${ }^{11}$ http://public.wiki.integro.geosys.ru/index.php/Категория:Руководство_пользователя

${ }^{12}$ https://ru.wikipedia.org/wiki/Описательная_статистика
} 
образов и компонентного анализа. Алгоритмы базируются на проверке многомерных статистических гипотез.

Таким образом, для «КОСКАД 3D» можно выделить следующие функциональные блоки: операции с данными («Сервис») и первичный анализ данных («Статистика»), прямые и обратные задачи, включая качественную интерпретацию («Фильтрация», «Обнаружение», «Статистика»), комплексная интерпретация («Комплекс»), визуализация («Графика»).

Дополнительно можно отметить: сайт программы имеет только русский вариант и не обновлялся с 2013 года; страница с демо-версией, дает ошибку 404; руководство пользователя датировано 2008 годом; на youtube.com нет канала, посвященного программе, ни авторских видео, ни видео пользователей, хотя только по данным авторов пакет используют в 52 крупных российских образовательных и производственных организациях.

Российский пакет СИГМА3D ${ }^{13}$ предназначен для структурной интерпретации гравитационных и магнитных аномалий, а также может быть использован для решения некоторых других задач (обнаружение объектов трубочного типа в том числе кимберлитовых и урановых; обнаружение следов миграции углеводородов по изменениям петрофизических характеристик горных пород). Разработка СИГМАЗD ведется более 20 лет.

Пакет является набором отдельных программ.

Программа REIST предназначена для построения модели субгоризонтального слоя с намагниченностью и плотностью, изменяющимися по латерали. Верхняя кромка слоя задается по геолого-геофизическим данным, а нижняя кромка, как правило, горизонтальная.

Программа DVOP предназначена для вычисления объемного распределения эффективной плотности и эффективной намагниченности.

B программе ROMGAS находятся координаты особых точек функций, описывающих магнитные и гравитационные аномалии.

В программе CLASS2 выполняется кластеризация пространственно распределенных данных.

Программа QUASI дает количественную оценку изолированных аномалий, используя подбор с помощью вертикального стержня.

Программа OPRES осуществляет вероятностное распознавание аномалий заданной формы.

В программе MGK по геофизическим данным картируются разрывные нарушения с использованием фильтрации данных методом главных компонент.

К числу дополнительных программ, не входящих в пакет, относятся.

Программа IGLA - количественная интерпретации аномалий с использованием модели усеченной пирамиды с горизонтальными верхней и нижней гранями.

B программе RELMASS - поправки за рельеф при расчете аномалий силы тяжести.

В программе СИНГУЛЯР - интерпретация методом особых точек.

Программа KORPRO - корреляционный метод разделения аномалий.

Программа PREDICTAR- прогноз целевого объекта по комплексу геологогеофизических данных.

B программе TRANSGRID - трансформации геофизических полей (линейная, нелинейная и специальная фильтрации, выделение градиентов)

Таким образом, в пакете СИГМА3D частично подготовку данных осуществляет программа RELMASS и, видимо, часть функций несет программа TRANSGRID, не входя-

\footnotetext{
${ }^{13} \mathrm{http}: / /$ sigma3d.com
} 
щая в пакет. Программа MGK осуществляет качественную интерпретацию. Решение обратных задач разнесено по семи программам: REIST, DVOP, ROMGAS, QUASI, IGLA, СИНГУЛЯР, KORPRO, причем три последних относятся к числу дополнительных. Две программы осуществляют классификацию: CLASS2, OPRES. Программа PREDICTAR осуществляет прогноз.

Дополнительно можно отметить: сайт программы де-факто имеет только русский вариант (английская версия пустая) и не обновлялся с 2016 года; на сайте имеются монографии авторов по интерпретации гравитационных и магнитных аномалий и журнальные статьи с примерами геологической интерпретации; демо-версий программ и руководств для пользователей нет в виду многочисленности и разноплановости программ; на youtube.com нет канала, посвященного пакету программ, хотя только по данным авторов пакет используют в 15 крупных российских производственных организациях; отдельные видео с примерами применения есть на канале общегеологической тематики $^{14}$, ведущемся совсем другой фирмой.

Российский пакет программ Zond ${ }^{15}$ предназначен для интерпретации электроразведки, сейсморазведки, магниторазведки и гравиразведки. Пакет разрабатывается с 2000 года.

Для гравиразведки и магниторазведки предназначены две программы - для профильной и площадной интерпретации: ZondGM2D и ZondGM3D.

Программа ZondGM2D предназначена для двумерной интерпретации данных наземных, скважинных и аэронаблюдений. В меню имеются следующие возможности: задание различных типов описаний модели среды с разной детальностью, вычитание нормального поля, введение поправок, качественная и количественная интерпретация. Интерфейс программы имеет русский и английский варианты.

Программа ZondGM3D предназначена для трехмерной интерпретации данных магниторазведки и гравиразведки в наземном и аэро вариантах. Интерфейс программы имеет только английский вариант, но во многом повторяет ZondGM2D, что позволяет быстро переключаться на работу с трехмерными задачами. Обе программы ZondGM2D и ZondGM3D выглядит лаконично, интерфейсы не перегружены. В указанных программах имеются почти все ранее упомянутые функциональные блоки: операции с данными, прямые и обратные задачи, включая качественную интерпретацию, визуализация. Отсутствуют возможности сложной комплексной интерпретации и детального статистического анализа исходных данных.

Дополнительно можно отметить: сайт программы имеет русский и полноценный английский вариант и регулярно обновляется; есть свежие демо-версии программ и руководства пользователей на русском и английском языках; имеются видео-уроки по программам пакета и примеры геологической интерпретации; есть раздел с информацией об услугах; автор с 2013 года ведет уoutube-канал ${ }^{16}$, где в видео информирует о последних изменениях в работе программ; программы используется в более 100 российских и зарубежных образовательных и производственных организациях.

Крупнейшим зарубежным пакетом, используемым в России, является Oasis Montaj, принадлежащий группе Seequent (с 1986 до ноября 2018 года - компании Geosoft ${ }^{17}$ ). B пакете имеются развитые возможности оперирования с данными, включая следующие. Операции с базами данных, в том числе выборки и различные манипуляции с данными

\footnotetext{
${ }^{14}$ https://www.youtube.com/c/ПервыйГеологический/videos

${ }^{15} \mathrm{http}: / /$ zond-geo.com

${ }^{16} \mathrm{https}: / /$ www.youtube.com/channel/UCGtprIIZkc9CsLfiuz4VvmQ

${ }^{17} \mathrm{https}$ ://www.seequent.com/seequent-acquires-geosoft
} 
[18]. Операции построения сеток различными методами и продвинутые возможности для работы с сетками и картами (математические преобразования, фильтрации и т.д). Поддерживается работа с более 40 различными форматами данных и изображений. Геолого-геофизическая функциональность вынесена в расширения, продаваемые отдельно.

Для геологического и геофизического анализа используются расширения [19]: геофизические (гравиметрия и магнитометрия, поправки за рельеф, электрометрия), геохимические, для бурения, аэрогеофизические, для дополнительных возможностей фильтрации и картографирования. Для гравиметрии и магнитометрии применяются двух- и трехмерное моделирование, а также методы трехмерной деконволюции Эйлера, расчета коэффициентов магнитной корреляции Китинга для поиска кимберлитов (метод распознавания образов), определения границ источника (анализ градиентов), расчет глубины залегания магнитных источников (аналог деконволюции Эйлера).

Пакет Oasis Montaj и его дополнения достаточно хорошо структурированы, имеются все ранее упомянутые функциональные блоки: операции с данными, прямые и обратные задачи, включая качественную интерпретацию, визуализация; возможности сложной комплексной интерпретации и детального статистического анализа исходных данных реализованы как операции с сетками.

Дополнительно можно отметить: сайт программы имеет английский и полноценный русский вариант и регулярно обновляется; после регистрации на сайте доступны свежие демо-версии программ, на английском языке имеется информация о релизах, руководство пользователя, обучающие видео, форум и многое другое, характерное для крупной международной компании. Общедоступный канал youtube содержит видео 2011-2017 годов ${ }^{18}$, являясь заброшенным после того как группа Seequent купила Geosoft, что, вероятно, связано с тем, что Seequent перемещает всю активность пользователей на свой сайт. Стоимость Oasis montaj Potential Fields Package составляет \$223 USD в день или \$1561 USD в неделю (цены доступны в личном кабинете после регистрации).

Рассмотрев пять самых популярных проприетарных геофизических пакета, используемых в России, содержащих программные средства обработки данных для гравиразведки и магниторазведки, можно отметить следующее.

Во-первых, различаются программы, предназначенные преимущественно для внутреннего российского употребления («КОСКАД 3D» и СИГМАЗD) и для международных продаж (ГИС INTEGRO, Zond и Oasis montaj). Различие касается в первую очередь удобства операций с данными, их форматирования и преобразования. Различия в функциональной наполненности менее значимы, но, тем не менее, присутствуют: у программ второй группы она гораздо менее дробная.

Во-вторых, различным является предел сложности, на котором останавливается развитие собственных программных средств. Для первой группы он достаточно низок, что обусловлено преимущественно внутрироссийским использованием, то во второй группе есть интересные нюансы. Если в ГИС INTEGRO и Zond все делается авторами, то в Oasis montaj даже в инсталляцию простейшего бесплатного Geosoft Viewer входит Python c предустановленными дополнительно пакетами для численных методов: numpy, pandas, vectormath, a также дополнительными пакетами для визуализации (steno3d), сети (isapi) и другими.

B-третьих, все программы имеют закрытый программный код, что делает пользователя полностью зависимым от собственника программы, что в сочетании с тенденцией

\footnotetext{
${ }^{18} \mathrm{https}: / /$ www.youtube.com/user/GeosoftTV/videos
} 
перехода проприетарного программного обеспечения на подписочную модель продаж представляется не самой лучшей перспективой. С другой стороны, стремление даже таких гигантов как Seequent использовать развитые программные средства свободного программного обеспечения показывает, что проприетарные программы являются не единственным способом выполнения сложных численных расчетов и построения развитых визуальных средств.

Остальные упомянутые выше проприетарные программы для интерпретации гравиразведки и магниторазведки (VECTOR, MultAlt), а также гравиразведки и сейморазведки (GCIS, GeoVIP) не имеют своих сайтов и известны только по страничке-анонсу на сайте организации-владельца (MultAlt ${ }^{9,19}$ ) и публикациям авторов. Вероятно, VECTOR, MultAlt попали в такое состояние потому, что сосредоточились в основном на геопотенциальных методах и не стали добавлять, как минимум, развитые возможности работы с данными и интерпретацию других геофизических методов.

Для гравиметрии и магнитометрии было показано, что использование свободного программного обеспечения (СПО) дает не меньшие возможности, что и проприетарного. При интерпретации геопотенциальных полей можно использовать следующие библиотеки и программы CПО: PyGIMLi, Fatiando a Terra, PyGMI, SimPEG [3].

Для удобства сравнения рассмотрим программу РyGMI ${ }^{20}$, которая имеет графический интерфейс, а не представляет собой просто библиотеку функций.

PyGMI является программой для моделирования и интерпретации, предназначенной для обработки электрометрических (включая магнитотеллурические), сейсмических, магнитометрических, гравиметрических, сейсмологических данных, а также данных дистанционного зондирования Земли. Программа разрабатывается с 2014 года.

Наиболее полно представлена обработка данных магнитометрии и гравиметрии, обработка других - фрагментарно и иногда основана на других открытых пакетах (электрометрия - библиотека SimPEG, магнитотеллурика - пакет MTру).

Рассмотрим блоки обработки программы PyGMI, связанные с интерпретацией магнитометрии и гравиметрии.

Исходные данные, графы их обработки и результаты объединяются в проект, который сохраняется в формате јson, для чтения которого есть функции во всех языках программирования. Данные гравиразведки и магниторазведки могут вводиться в виде растров, векторов или в виде файлов, создаваемых гравиметром CG-5.

Под растровыми данными понимаются как непосредственно изображения (в форматах: GeoTIFF $^{21}$ - растр TIFF совместно с метаданными о географической привязке; ERDAS IMAGINE File Format ${ }^{22}$ (IMG) - многослойные растровые изображения с географической привязкой для обработки данных дистанционного зондирования), так и точечные данные в формате ASCII XYZ и сетки различных форматов, генерируемые программами (Surfer grid, SAGA grid, GeoPak grid, ArcInfo Binary Grid и др.).

Для растровых данных (меню «Raster») применяются следующие обработки: по задаваемым формулам и алгоритмам, включая объединение данных и замену данных и пропусков; нормализация различными способами; сглаживание с разной формой окна; вычисление горизонтальных и вертикальных градиентов; продолжение поля вверх и вниз; пересчет сеток с другим шагом; трансформация из одной картографической проекции в другую; обрезка (бланкирование) карт с использованием полигонов, загружае-

\footnotetext{
${ }^{19} \mathrm{https}$ ://rosgeo.com/services/intellektualnye-uslugi/it-tekhnologii-i-servisy/tekhnologiyamnogoalternativnoy-klassifikatsii-i-prognoza-multalt

${ }^{20} \mathrm{https}$ ://patrick-cole.github.io/pygmi

${ }^{21} \mathrm{https}: / /$ ru.wikipedia.org/wiki/GeoTIFF

${ }^{22} \mathrm{https} / / /$ www.loc.gov/preservation/digital/formats/fdd/fdd000420.shtml
} 
мых из shp-файлов; выделение профилей из сеточных данных. Имеются развитые визуализации сеточных данных с сохранением результата в файл формата GeoTIFF.

Для векторных данных (меню «Vector») возможен ввод shp-файлов, а также точечных и линейных данных. Трансформация данных возможна путем: удаления лишних точек с использованием полигонов; перевода в другую систему координат или картографическую проекцию; пересчета на сетку точечных или линейных данных.

Скважинные данные (меню «Boreholes») вводятся из файлов электронных таблиц Excel или текстовых (csv) форматов. Выполняется визуализация данных с отображением наклона, литологии, стратиграфии, а также координат скважины, даты бурения и другой дополнительной информации.

В разделе классификация (меню «Classification») выполняются кластерный анализ и сегментация изображений, которые могут выполнять автоматическую интерпретацию растровых многомерных данных. При кластерном анализе возможно задание: масштабирования данных как операции, предваряющей непосредственно кластерный анализ, например удаление среднего и масштабирование до единичной дисперсии; методов кластеризации $\left(\mathrm{k}-\right.$ средних $\left.^{23}, \mathrm{DBSCAN}^{24}, \mathrm{BIRCH}^{25}\right)$, параметров метода, например, для k-средних: минимального и максимального числа кластеров, максимального числа итераций, точность; выполнение четкой и нечеткой кластеризации. Сегментация изображений выполняется по методике Баатца и Шепе [16]. Еще одна возможность - классификация с учителем, когда пользователь определяет классы, добавляя многоугольники на карту. Также имеется визуализации результатов кластерного анализа как на картах, так и на диаграммах рассеяния.

В разделе «Гравиметрия» (меню «Gravity») есть возможность ввода файлов гравиметров CG-5 и файлов GPS с заданием при необходимости дополнительных параметров для привязки. Имеется возможность обработки данных в соответствии с североамериканским стандартом [17]. Команда «Обработка» выполняет привязку данных к заданным опорным точкам.

В разделе «Магнитометрия» (меню «Magnetics») вычисляются: международное геомагнитное аналитическое поле ${ }^{26}$, приведение к полюсу, угол наклона магнитного поля для сглаживания данных, расчета глубин до тел с вертикальными стенками по углу наклона магнитного поля.

Программа PyGMI хорошо структурирована, имеются все ранее упомянутые функциональные блоки: операции с данными, включая детальный статистический анализ исходных данных, обратные задачи (магнитометрия), включая качественную интерпретацию, визуализация. Для гравиметрии практически нет собственных средств в PyGMI, но это может быть компенсировано использованием других свободных пакетов, например, SymPEG или PyGIMLi.

Дополнительно можно отметить: сайт регулярно обновляется, имеются руководство пользователя и обучающее видео. На уoutube есть одно видео ${ }^{27}$ с авторским рассказом о программе.

\section{Выводы}

Проанализированы вопросы функциональности и развития программного обеспечения используемого для решения задач гравиметрии и магнитометрии. Выделены ос-

\footnotetext{
${ }^{23}$ https://ru.wikipedia.org/wiki/Метод_k-средних

${ }^{24} \mathrm{https}$ ://ru.wikipedia.org/wiki/DBSCĀN

${ }^{25} \mathrm{https} / / /$ ru.wikipedia.org/wiki/BIRCH

${ }^{26} \mathrm{https}$ ://ru.wikipedia.org/wiki/Международное_геомагнитное_аналитическое_поле

${ }^{27} \mathrm{https}: / / \mathrm{www}$. youtube.com/watch? $=5 \mathrm{kM} 3 \mathrm{tKkjoSw}$
} 
новные программные блоки: операции с данными, первичный анализ, качественная и количественная интерпретация, комплексная интерпретация, визуализация.

Детально рассмотрены проприетарные программы интерпретации геопотенциальных полей, используемые в России (ГИС INTEGRO, «КОСКАД 3D», СИГMA3D, Zond, Oasis Montaj). Указанное программное обеспечение достаточно четко отображает цели разработчиков. Если целью было преимущественно внутреннее использование, то с одной стороны некоторые интерпретационные алгоритмы хорошо проработаны, а с другой стороны сервисная часть оставляет желать лучшего: или слабая сервисная часть (структурирование, повтор функциональности, операции с данными не проработаны, нет описаний) или программы медленно развиваются, несмотря на солидный возраст. Если целью с самого начала были продажи, то сервисная и информационная составляющие хорошо проработаны, но меньше разнообразие алгоритмов интерпретации. Значащим вопросом является цена: за умеренную цену можно получить только умеренные возможности, большие возможности можно получить только за большую цену. Все это заставляет обращаться для решения задач гравиметрии и магнитометрии к свободному программному обеспечению.

Выполнено подробное описание программы PyGMI, имеющей графический интерфейс пользователя и обеспечивающей решение различные геологогеофизических задач, начиная от работы со скважинными данными, до решения задач полевой геофизики и дистанционного зондирования Земли. Для решения задач магнитометрии и, в меньшей степени, гравиметрии имеется широкий спектр возможностей: манипуляции с данными, классификации разными методами, качественная и количественная интерпретация при решении обратных задач. Преимущество открытого программного обеспечения связано с возможностью исследовать и модифицировать программный код под свои нужды, а также использовать другие свободные библиотеки для получения дополнительных функций. Важной особенностью свободного ПО является возможность сотрудничать в своей научной области с другими заинтересованными разработчиками по всему миру, обмениваясь передовыми разработками, обеспечивая современность и актуальность программ.

\section{БИБЛИОГРАФИЧЕСКИЙ СПИСОК}

1. Булах Е.Г., Ржаницын В.А., Маркова М.Н. Применение метода минимизации для решения задач структурной геологии по данным гравиразведки. - Киев: Наук. думка, 1976. - 219 с.: ил.

2. Вельтистова О.М., Мотрюк Е.Н. Современные отечественные программные комплексы интерпретации гравимагнитных данных // Изв. Коми науч. центра. - 2013. - № 3 (15). - С .70-80. - URL: https://cyberleninka.ru/article/n/sovremennye-otechestvennye-programmnye-kompleksy-interpretatsiigravimagnitnyh-dannyh (дата обращения 17.05.2021).

3. Геник И.В. Свободное программное обеспечение для задач гравиразведки и магниторазведки // Горное эхо. - 2020. - № 3 (80). - С. 60-66. - DOI: 10.7242/echo.2020.3.12.

4. Гольцман Ф.М., Калинин Д.Ф., Калинина Т.Б. Компьютерная технология MultAlt альтернативной классификации и прогноза по комплексу геоданных // Российский геофизический журнал. - 2000. № 17-18. - С. 64-70.

5. Давыденко А.Ю. Интерпретация геофизических данных с использованием технологии GelioSMI // Вопросы естествознания. - 2016. - № 1(9). - С. 120-124.

6. Калинин Д.Ф. Компьютерная технология статистической многоальтернативной комплексной интерпретации для решения прогнозно-поисковых задач рудной геофизики: дис. ... д.т.н.: 25.00.35. - М., 2005. - 346 с.: ил.

7. Кобрунов А.И., Куделин С.Г., Мотрюк Е.Н. Интегрированная среда физико-геологического моделирования на основе системной инверсии [Электронное издание] / УГТУ. - Ухта, 2015. - URL: https://www.geokniga.org/bookfiles/geokniga-integrirovannaya-sreda-fiziko-geologicheskogomodelirovaniya-na-osnove-sistemn.pdf (дата обращения 17.05.2021). 
8. Куделин С.Г., Барабанов М.И., Кобрунов А.И. Программный редактор "GeoVIP" как компонент среды физико-геологического моделирования на основе системной инверсии // Информационные технологии в управлении и экономике. - 2012. - №3 (03). - C. 18-26. - URL:

https://elibrary.ru/item.asp?id=26236619 (дата обращения 17.05.2021).

9. Логачев А.А., Захаров В.П. Магниторазведка. - 5-е изд., перераб. и доп. - Л.: Недра, 1979. - 351 с.: ил.

10. 10.Матусевич А.В. Гравиразведка Прикаспийской впадины [Электронное издание]. - Ливны: Издатель Мухаметов Г.В., 2013. - 176 с. - URL:

http://www.academy.kz/images/files/matusevich/gravirazvedka.pdf. (дата обращения 17.05.2021).

11. Немцов Л.Д. Высокоточная гравиразведка. - М.: Недра, 1967. - 230 с.

12. Никитин А.А., Булычев А.А. Комплексный анализ и комплексная интерпретация геофизических полей: учеб. пособие. - М., ВНИИгеосистем, 2015. - 93 с.: ил. - URL: http://geophys.geol.msu.ru/Komplex.pdf (дата обращения 17.05.2021).

13. Программа интерпретации геопотенциальных полей методом векторного сканирования VECTOR: свидетельство об офиц. регистрации № 2004611611 / Новоселицкий В.М., Кутин В.А., Чадаев М.С., Бычков С.Г., Простолупов Г.В., Антипин В.В.; заявитель и правообладатель ГИ УрО РАН. - № 2004610697; заявл. 29.03.04; зарегистрировано 01.07.04.

14. Старостенко В.И. Устойчивые численные методы в задачах гравиметрии. - Киев, Наук. думка, 1978. 227 с.: ил.

15. Шванк О.А., Люстих Е.Н. Интерпретация гравитационных наблюдений: Теория и практика решения прямой и обратной задачи гравиметр. разведки. - М.; Л.: Гостоптехиздат, 1947. - 400 с.: черт.

16. Baatz M., Schape A. Multiresolution Segmentation: An Optimization Approach for High Quality MultiScale Image Segmentation. // Angewandte Geographische Informations-Verarbeitung XII / eds.: Strobl J., Blaschke T., Griesbner G. - Wichmann Verlag, 2000. - 542 p.

17. Hinze W.J., Aiken C., Brozena J., Coakley B., Dater D., Flanagan G., Forsberg R., Hildenbrand T., Keller G.R., Kellogg J., Kucks R., Li X., Mainville A., Moring R., Pilkington M., Plouff D., Ravat D., Roman D., Urrutia-Fucugauchi J., Veronneau M., Webring M., Winester D. New standards for reducing gravity data: the North American gravity database // Geophysics. - 2005. - V. 70, 4. - P. J25-J32. - DOI: $101190 / 1 / 1988183$.

18. Oasis montaj: Программное обеспечение для обработки данных и построения карт. - Geosoft, 2012.

19. Oasis montaj: Software for Earth Science Mapping and Processing. - Geosoft, 2012.

УДК 550.8.05

DOI:10.7242/echo.2021.2.11

\title{
КОЛИЧЕСТВЕННЫЙ АНАЛИЗ ИНТЕРФЕРЕНЦИОННЫХ ЭФФЕКТОВ В ШАХТНОЙ СЕЙСМОРАЗВЕДКЕ
}

\author{
И.А. Санфиров ${ }^{1}$, Т.В. Байбакова ${ }^{1}$, С.А. Миронов ${ }^{2}$ \\ ${ }^{I}$ Горный институт УрО РАН, г. Пермь \\ ${ }^{2}$ ПАО «Уралкалий»
}

\begin{abstract}
Аннотация. Выбран тестовый участок шахтного поля соляного рудника с известными особенностями геологического строения. Выполнены наземные и шахтные сейсморазведочные исследования. Определены особенности волновых полей, обусловленные геологическим строением тестового участка. Реализована «специальная» шахтная сейсморазведочная технология, инициирующая интерференционное волновое поле. Оценены искажения волновых параметров за счет подобных эффектов, влияющие на формирование интерпретационных заключений.
\end{abstract}

Ключевые слова: шахтная сейсморазведка, отраженные волны, интерференционные явления, поперечные волны, продольные волны, интерпретационные искажения.

При интерпретационных построениях по результатам цифровой обработки сейсморазведочных данных необходим учет разного рода интерференционных эффектов, вызванных тонкослоистым строением реальных сред. Вероятность их проявления оценивается по результатам спектрального анализа сейсмических записей, которые, как известно, в частотной области представляют собой произведение ампли- 